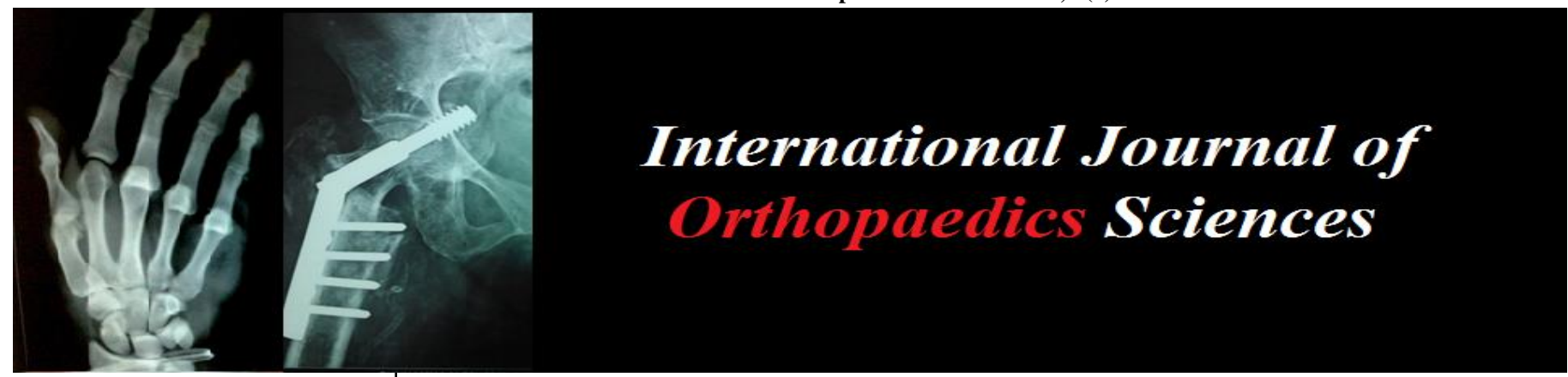

ISSN: $2395-1958$

IJOS 2018; 4(4): 605-608

(C) 2018 IJOS

www.orthopaper.com

Received: 19-08-2018

Accepted: 20-09-2018

Vijay Chandru

Department of Orthopaedics,

JSS Hospital, Mahatma Gandhi

Road, Mysore, Karnataka, India

Ravikiran HG

Department of Orthopaedics,

JSS Hospital, Mahatma Gandhi

Road, Mysore, Karnataka, India

Sumith Raj

Department of Orthopaedics,

JSS Hospital, Mahatma Gandhi

Road, Mysore, Karnataka, India

Correspondence

Vijay Chandru

Department of Orthopaedics,

JSS Hospital, Mahatma Gandhi

Road, Mysore, Karnataka, India

\section{To compare and analyze the functional outcome seen after hemiarthroplasty with uncemented Austin Moore's prosthesis and non-cemented non-modular bipolar prosthesis in all displaced intracapsular femur neck fractures in elderly patients using the Harris Hip score in rural population}

\author{
Vijay Chandru, Ravikiran HG and Sumith Raj
}

DOI: https://doi.org/10.22271/ortho.2018.v4.i4g.69

\begin{abstract}
Introduction: Fractures Neck of the femur is one of most common fractures seen by an orthopedician. The incidence of these fractures and the various problems arising from them are growing, the reason for this is mainly the increase in the occurrence of osteoporosis in elderly population.

Aims and Objectives: To compare and analyze the functional outcome seen after hemiarthroplasty in displaced intracapsular fracture neck of the femur elderly patients using uncemented Austin Moore's prosthesis and uncemented non-modular bipolar prosthesis by using Harris Hip score system in rural population.

Observations and Results: In the present study total number of cases registered is 52 and one patient from both groups could not complete the study due to myocardial infarction, male and female ratio was 24/28. Out of 52 patient, the youngest patient among these group is 60years and oldest among this group is 90 years. The average age of the patient in group A is 72.68 years in Group B is 70 years. All the patients were encouraged to ambulate after 3 days after the surgery. All the 50 patients were followed up for minimum period of one year. Loosening, erosions or deep infections did not occur in both the group until the last follow up in our study.

Discussion: In the present study it was proved that femoral neck fractures in old patient causes severe problems both to patient as well as to society. In a present scenario, hemiarthroplasties are limitedly used for selective patients.

Conclusion: Primary Hemiarthroplasty, it is an effective surgery for most of the neck fractures in old age patients. The success rate of this surgery mainly depends on proper planning and execution to get best outcome. One year later percentage of patients achieving good to excellent results and the functional outcome with non-modular uncemented bipolar prosthesis were more than those with unipolar Austin Moore's prosthesis though it was not statistically significant.
\end{abstract}

Keywords: Fractures neck femur, unipolar Austin Moore's prosthesis, non-cemented non-modular bipolar prosthesis, Harris Hip score, hemiarthroplasty

\section{Introduction}

Fractures neck of the femur are the common fractures seen by an orthopedician. As the incidence and prevalence of the osteoporosis is increasing so as the fracture neck of the femur and problems arising secondary to fracture neck of the femur. Because of the lack of cambium layer fracture bathed in synovial fluid and tamponade effect of the hematoma formed in the intracapsular region which leads to nonunion, malunion and increased chances of avascular necrosis of the head of the femur. Primary hemiarthroplasty or the total hip arthroplasty is preferred in elderly patients. Nonunion malunion and avascular necrosis of the head of the femur will increase in displaced fracture neck of the femur ${ }^{[1,2]}$. Primary arthroplasty or the total hip arthroplasty is the treatment of choice for all displaced fractures neck of the femur in elderly patients. Unipolar hemiarthroplasty or bipolar hemiarthroplasty can be done instead of total hip arthroplasty for the displaced fracture neck of the femur with lesser morbidity and 
minimal operating time especially in patients not willing for total hip athrroplasty commonly because of the people arriving from rural areas and belong to the low socioeconomic class hemiarthroplsty was done by Moore and Bohlman in 1943 mainly to replace the head of the femur in proximal femur tumor with metallic head and neck prosthesis ${ }^{[3]}$. Later Moores prosthesis and Thopmsons prosthesis were commercially available to treat fracture neck of the femur in neurologically handicapped and risk is high for the patients. Unipolar prosthesis relatively good results and led to erosion of the acetabulum joint pain protrusio acetabuli then in 1974 James introduced bipolar prosthesis where in above complications relatively decreased.

\section{Aims and objectives}

To anlyse and compare the functional outcome seen after hemiarthroplasty in displaced neck of the femur in elderly patients using either non-cemented Austin Moore's prosthesis or non-cemented non-modular bipolar prosthesis with the use of Harris Hip score system for patients hailing from rural population area.

\section{Materials and methods}

It is a prospective study consists of all the patients admitted to JSS hospital with intracapsular fracture neck femur treated with unipolar or bipolar endoprosthesis.

\section{Inclusion Criteria:}

1. Male and female patients aged more than 50 years

2. Displaced fracture neck of femur

3. Old and Neglected cases of fracture neck of femur

4. Fracture neck of femur with nonunion

Sample size: All patients undergoing hemiarthroplasties were divided in two groups, group A being treated with unipolar and group B with bipolar endoprosthesis. Each group had 26 patients.

We selected patients for uncemented Austin Moore's prosthesis and non-cemented non-modular bipolar prosthesis depending mainly on the age criteria and patients co morbidities and the physiological age of the patient and preoperative ambulatory status of the patient prior to the history of fall. keeping above factors in mind we did uncemented Austin Moore's prosthesis if the physiological age is more and above 70 years with low preoperative ambulatory status and if the patient is suffering other co morbidities like diabetes hypertension and if any cardiac issues with less ejection fraction where as we opted uncemented bipolar prosthesis if the physiological age is less and less than 60 years and no co morbidities keeping in mind that they may need revision with total hip arthroplasty if they any complication occur such protrsusio acetabuli as the chances of this complication is less with bipolar prosthesis as this would improve the quality of life. the patients were subjected to the hemiarthroplasty after getting the fitness surgery with southern moores approach and head of the femur extracted and joint inspected for any osteoarthritis changes after broaching and preparing the femur implant was put and stability was checked later closure the wound done with drain.

\section{Observations and results}

Out of 52 patient, the youngest patient among these group is $60 y e a r s$ and oldest among this group is 90 years. The average age of the patient in group A is 72.68 years in Group B is 70 years. All the patients were encouraged to ambulate after 3 days after the surgery. All the 50 patients were followed up for minimum period of one year. Loosening, erosions or deep infections did not occur in both the group until the last follow up in our study.

In this study $79 \%$ of the patients had good to excellent outcome in the age group of 60-64 in group A and in group B $100 \%$ of the cases had good to excellent results where as in the Age group of $65-69,83 \%$ of the cases had good to Excellent results in Group A when compared to $100 \%$ in group B. patients belonging to the of 70- 74 years, in group A had $79 \%$ had good to excellent outcome which is compared to $100 \%$ in Group B. Between the age group of $75-79,78 \%$ had good to excellent results when compared to $65 \%$ in group B which less when compared to group A. whereas above 80 age group $70 \%$ of the cases in Group A had good to excellent results which was is more when compared to 30\%in group B. Assessment of Functional Outcome after Clinico-radiological assessment and measurement according to Harris Hip Score was done in this study at following intervals. Follow up after 6 weeks, mean functional core in Group A was 62 which as compared to 63 in Group B which is statistically insignificant At 12 weeks of follow up, mean functional score in Group A is 78.1, compared to 79 in Group B which is statistically insignificant. Mean functional score increased among both in group A and Group B which was 83 and 85 respectively which is statistically not significant.

At the end of 1 year mean functional score in group A is 89 when compared to 93 in group B patients.

In all the operated patients, medial offset and limb length discrepancy was ascertained postoperatively using PACS. Medial Offset difference of less than $5 \mathrm{~mm}$ was considered as optimal. In Group A, i.e. the unipolar group, the mean medial offset difference was observed to be 2.1 as compared to Group B where the value was 1.4 which was not statistically insignificant. In Group A, of the 25 patients, 10 patients had negative medial offset whereas 15 patients had positive medial offset. As compared to the Group B, there were 4 patients with negative medial offset and 21 patients with positive medial offset. In Group A, optimal Medial offset was seen in 20 patients and 5 patients were suboptimal. In Group B, optimal medial offset difference was seen 24 and suboptimal in 1 patient.

Limb length discrepancy of less than $10 \mathrm{~mm}$ was considered to be suboptimal. In Group A, the unipolar group, the mean limb length discrepancy is $4.5 \mathrm{~mm}$ when compared to $3.9 \mathrm{~mm}$ in Group B which was found to be statistically not significant. Shortening was seen in 11 patients and lengthening in 14 patients in group A. Optimal limb length discrepancy was noticed in 22 patients. Where as in Group B, shortening was seen in 2 patients and lengthening in 23 patients. Optimal limb length discrepancy was noticed in 23 patients.

\section{Discussion}

In the present study it was proved that femoral neck fractures in old patient causes severe problems both to patient as well as to society. In a present scenario, hemiarthroplasties are being used limitedly for selective patients. While many studies have examined the role of hip biomechanics in the setting of elective hip arthroplasty, the trauma literature is scant. osteoporosis is common elderly patients which poses problem while fixing the fracture with the implants as there is limited bone sock and the purchase of the implant is not good requiring prolonged immobilization which leads to implant failure and thus nonunion. Early ambulation with weight bearing is generally required keep the fit as their general condition is compromised because of the age and associated 
diseases. The rate of reoperation in hemiarthroplasty is only 10 to $18 \%$ when compared with fracture fixation with internal fixation where the rate of reoperation is 34 to $36 \%$ and better functional outcome is achieved with primary hemiarthroplasty than with fracture fixation. Hemiarthroplasty provides a much shorter procedure along with cost effectiveness especially suited for Indian rural population as vast majority of the population belong to the low socioeconomic class and not willing for total hip arthroplasty, which comprise of highly osteoporotic elderly individuals with shorter life expectancy $[4,5]$.

This study is a prospective and comparative study of hemiarthroplasties treated with either unipolar Austin Moore's prosthesis or with nonmodular bipolar prosthesis as the surgical modality of treatment for a displaced fracture neck of the femur in elderly patients. In our study we used the Harris Hip Score as a basis of functional outcome assessment. Our study includes all the important variables displaying into single figure which is reliable, reproducible and objective reasonably. In our present study the mean age in Group A was 73 years and Group B was 70 years as compared to study done by Nottage et al it was 65 years for bipolar prosthesis and 73 years for the Austin Moores prosthesis their mean harris hip score of 85 in case of non-modular bipolar hemiarthroplasty and 77 in cases operated with unipolar Austin Moore's prosthesis with p value being insignificant ${ }^{[6]}$. Analysis done at the end of 2 months $48 \%$ of the patients in group A had well to excellent results which is same as $48 \%$ in group B, the difference is insignificant. analysis done at the end of 6 months after the surgery $76 \%$ of the patients in group $\mathrm{B}$ had good to excellent results when compared to $56 \%$ in group A which is statistically significant $.88 \%$ of the patients in Group B had excellent to good functional outcome which is more when compared to $76 \%$ of the cases in Group A at the end of 1 year. Again this difference is not statistically significant. Lesser percentage of outcome in group A compared to group B at 6 months followup may be because of complications related to Austin Moore's prosthesis.

Cornell et al. prospective study with 33 cases of non-modular hemiarthroplasty and 15 unipolar hemiarthroplasty with 6 months follow up also says that functional recovery and postoperative complication rates were insignificant between the two groups.

In our prospective study 1 patient operated with Austin Moore's hemiarthroplasty and 1 patient operated with bipolar prosthesis hemiarthroplasty died by 1 year follow up due to the is preopeartve medical comorbidities. Our study mortality rate is less because of the very good management of the medical problems treated preoperatively and also postoperatively, proper usage of antibiotics and most importantly early ambulation. Superficial infection rates is statistically insignificant between both the groups. Infection rates are less because of the strict prophylactic antibiotic protocol. The infection rate was little more if it operated using southern moores posterior approach for the hip because of the proximity of the incision to the perineal region. Patient with postoperative infections stayed in the hospital for little longer period. Infections were treated with regular dressings and antibiotics as per the antibiogram. We did not encounter any deep infection of the wound in our prospective study ${ }^{[7]}$.

Sciatic nerve injuries can occur because of the excessive traction retraction of the nerve and position of the limb while operating in about 0.7 to $3.5 \%$. We did not encounter any sciatic nerve injuries as surgery was done meticulously and proper retraction of the soft tissue and sciatic nerve. There were similar rates of dislocation both in unipolar and bipolar groups where as in our study we did not encounter not even a single case of dislocation.

In our prospective study, patient functional outcomes at the end of 1year are similar with that of the previous studies in the literature. Rehabilitation was good in our elder patients after the surgery for the femoral neck fractures as their medical and mental status was good prior to and also postsurgery. Postoperatively if the joint is stable pain less and achieving good range of movement those cases are successful in orthopedic perspective.

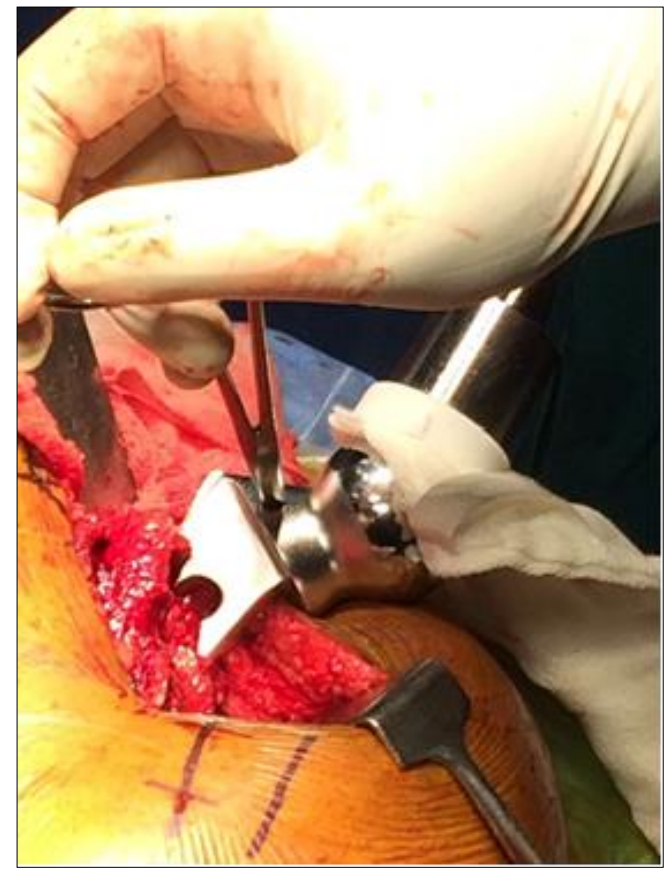

Photo 1: Austin Moore Prosthesis being inserted maintaining anteversion.

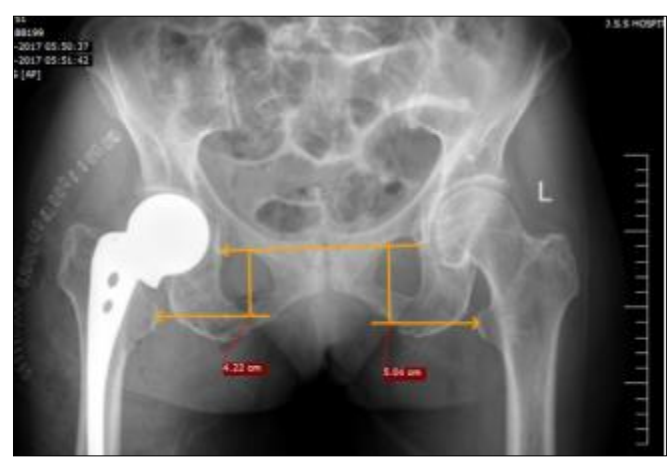

Photo 2: Measurement of limb length discrepancy in Austin Moores prosthesis.

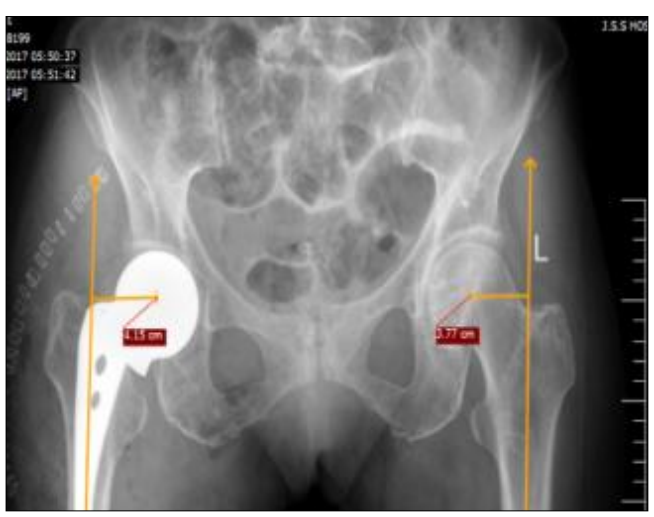

Photo 3: Medial offset measurement in Austin Moores prosthesis. 


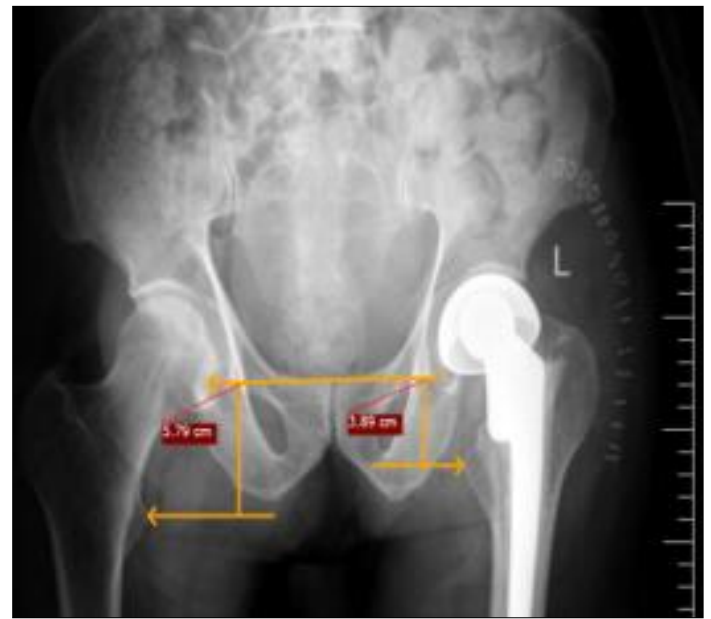

Photo 4: Limb Length Discrepancy measurement in bipolar prosthesis

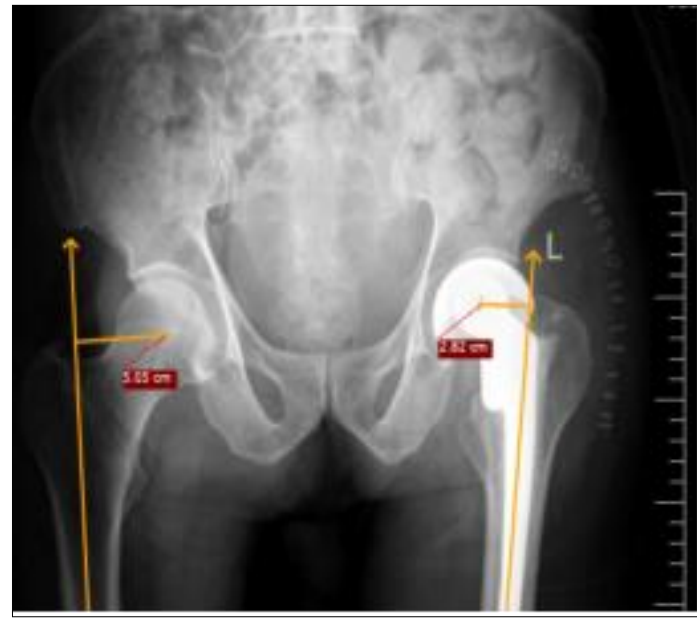

Photo 5: Measurement of the Offset in bipolar prosthesis

\section{Conclusion}

Hemiarthroplasty with unipolar Austin Moore prosthesis and non-modular bipolar prosthesis is commonly done and an effective surgery for most of the displaced fracture neck of the femur in elderly patients. Success of the surgery always depends on the preoperative planning, proper execution of the planning surgical techniques and postoperative protocol and rehabilitation therapy for the best results and outcome. the percentage of the patients achieving good to excellent results with bipolar prosthesis were more than that of the Austin Moores prosthesis even though it is statistically insignificant. by the end of one year mean Harris hip score was 89.5 in group A whereas group B it was 91.1. The outcome between unipolar and bipolar prosthesis is statistically insignificant, but in older patients unipolar prosthesis had better outcome as the longevity of the patient was less where as in uncemented bipolar group since the longevity of the patient was more had some complications later and the patient may have progressive senile osteoporosis if nutrition is not proper with little sunlight exposure. In Indian scenario where cost of treatment forms a major consideration before any procedure unipolar prosthesis provides with a easier and cost effective mode of treating displaced fracture neck of femur in elderly patients. Achieving medial offset and proper limb length postoperatively although is stressed upon in theory we did not find statistically significant difference. But limb length discrepancy above $10 \mathrm{~mm}$ was associated with poorer outcomes although it is statistically insignificant.

\section{References}

1. Wathne RA, Koval KJ, Aharonoff GB et al. Modular unipolar versus bipolar prosthesis: A prospective evaluation of functional outcome after femoral neck fracture. J Orthop Trauma. 1995; 9:298-302.

2. James Harkess. Campbell's operative orthopaedics. 10th edition. 2003; 1:315.

3. Narayan KK, George T. Functional outcome of fracture neck of femur treated with total hip replacement versus bipolar arthroplasty in a South Asian population. Arch Orthop Trauma Surg. 2006; 126(8):545-8.

4. Klestil $\mathrm{T}$ et al. Cementlesshemiarthroplasty in femoral neck fractures: evaluation of clinical results and measurement of migration by EBRA-FCA. Archives of orthopaedic and Trauma surgery. 2006; 126(6):380-386.

5. Chandran $\mathrm{P}$ et al. Mid term results of Furlong LOL uncemented hip hemiarthroplasty for fractures of the femoral neck. ActaOrthopBela. 2006; 72(4):428-433.

6. Nottage McMaster et al. Comparison of bipolar implants with fixed neck prostheses in femoral neck fractures. Corr. 1990; 251:38-43.

7. Ahmad I. Mortality and morbidity in elderly patients with fracture neck of femur treated by Hemiarthroplasty. J Coll Physicians Surg Pak. 2006; 16(10):655-8. 